\title{
Narrativa
}

\section{Classici italiani nel mondo. Quale canone per il nuovo millennio?}

Laura Di Nicola

\section{(2) OpenEdition}

1 Journals

\section{Edizione digitale}

URL: https://journals.openedition.org/narrativa/793

DOI: $10.4000 /$ narrativa.793

ISSN: 2804-1224

\section{Editore}

Presses universitaires de Paris Nanterre

\section{Edizione cartacea}

Data di pubblicazione: 1 décembre 2016

Paginazione: 43-54

ISBN: 978-2-84016-266-7

ISSN: $1166-3243$

\section{Notizia bibliografica digitale}

Laura Di Nicola, «Classici italiani nel mondo. Quale canone per il nuovo millennio?», Narrativa [Online], 38 | 2016, online dal 01 janvier 2022, consultato il 22 février 2022. URL: http://

journals.openedition.org/narrativa/793 ; DOI: https://doi.org/10.4000/narrativa.793

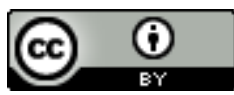

Narrativa est mise à disposition selon les termes de la Licence Creative Commons Attribution 4.0 International. 


\section{Classici italiani nel mondo. Quale canone per il nuovo millennio?}

La memoria conta veramente - per gli individui, le collettività, le civiltà - solo se tiene insieme l'impronta del passato e il progetto del futuro, se permette di fare senza dimenticare quel che si voleva fare, di diventare senza smettere di essere, di essere senza smettere di diventare (Italo Calvino).

distanza di poco oltre quindici anni dall'inizio del nuovo millennio, in un contesto in cui la letteratura sembra dover riconquistare la sua funzione sociale e il libro prefigura prospettive incerte, parlare di "canone per il nuovo millennio" dell'Italia fuori Italia pone sin da subito l'argomento in un orizzonte di riflessioni confuse, indefinite, provvisorie. Quali sono le autrici e gli autori di oggi che rappresenteranno i "classici" e le "classiche" della tradizione letteraria italiana del nostro millennio e del suo futuro canone? Come potrà definirsi una nuova, possibile, tradizione letteraria? Che ruolo avranno (anche per poter rispondere a tali domande) la critica e la storiografia letterarie, di cui è ormai dichiarata la crisi definitiva?

Si tratta di questioni che non si possono prevedere e che prefigurano anche possibili derive dello statuto dell'italianistica, italiana e straniera, della contemporaneistica, nei sistemi della ricerca attuale. Le controverse definizioni di "canone" e di "classico" pongono prospettive teoriche e metodologiche già complesse dentro l'Italia che diventano ancor più difficili da esplorare nel confronto con il fuori Italia.

Il rapporto fra prospettiva nazionale e sovranazionale allarga, infatti, gli interrogativi al concetto stesso di opera, di autore, di traduzione, di edizione. All'estero gli autori italiani sono decontestualizzati, o contestualizzati con criteri editoriali più che letterari. Le opere (soprattutto le raccolte di racconti o di poe- 
sie) subiscono rinascite. I titoli cambiano. Le traduzioni, pur con le loro trasformazioni, cominciano, tuttavia, a integrarsi "nel sistema della cultura ospite, che si rifrange sulla cultura d'origine" . Le edizioni estere presentano vesti e paratesti diversi. La letteratura italiana nel mondo si delinea, quindi, non tanto nel dibattito dell'italianistica internazionale, quanto dentro confini costruiti intorno alle politiche editoriali mondiali, fra gli agenti letterari, i mediatori culturali, i traduttori, figure in ombra protagoniste di un dialogo serrato fra identità culturali diverse.

Nel confronto interdisciplinare fra le storie dell'editoria internazionale, le teorie della traduzione, le storie letterarie, italiane, europee, mondiali, e la filologia dei testi, e nei reciproci sconfinamenti, si situano le premesse necessarie per affrontare un quadro ancora tutto da ricostruire, per l'assenza di strumenti, di bibliografie sistematiche, di luoghi della ricerca che non siano disseminati o virtuali. Da questo punto di vista è centrale il problema delle fonti per poter studiare $i$ classici italiani nel mondo, fonti bibliografiche, archivistiche e librarie, nazionali e internazionali, che conservano le tracce di un vitale rispecchiamento fra culture e lingue, impronte per risalire a una nuova storia dell'intellettualità italiana nomade e cosmopolita, che la storiografia letteraria e la filologia novecentesche hanno appena cominciato a prendere in considerazione per costruire una nuova idea di modernità letteraria. Vanno considerate in Italia le "biblioteche d'autore" (che conservano le opere in traduzione; ma anche possibili edizioni in italiano con annotazioni, tagli, interventi, apportati in previsione della preparazione di una traduzione), gli archivi degli scrittori (per le corrispondenze fra autore e traduttore, i contratti, le cifre di vendita), gli archivi e le biblioteche editoriali e degli agenti letterari ${ }^{2}$. Queste fonti si completano all'estero, con uno sguardo rovesciato, esplorando gli archivi e le biblioteche delle traduttrici e dei traduttori, delle case editrici, degli istituti italiani di cultura (da cui sono passate generazioni di scrittori), delle biblioteche nazionali mondiali. Consultando i cataloghi delle biblioteche nazionali mondiali emerge un patrimonio sterminato di opere in lingua originale o in traduzione di autori italiani, che costituisce

1. D’Intino, Franco, "Il Novecento italiano oltrefrontiera", in Borseldino, Nino, Felici, Lucio (a cura di), Storia della letteratura italiana. XI: Il Novecento. Scenari di fine secolo 1, Milano, Garzanti, 2001, p. 923.

2. Si veda FinocCHI, Luisa, "Il ruolo della mediazione editoriale. Le fonti", in DI Nicola, Laura, Schwartz, Cecilia, Libri in viaggio. Classici italiani in Svezia, Stockholm University, Acta Universitatis Stockholmiensis, 2013, pp. 100-106. 
un'ampia raccolta di una ideale bibliografia delle opere italiane tradotte in altre lingue, ma disseminato nei più vari paesi ${ }^{3}$.

Una mappa che delinei, in ambito bibliografico, biblioteconomico e archivistico, la circolazione, la diffusione, la ricezione, più complessivamente la fortuna, di autori e opere italiani all'estero va ancora costruita attraverso una rete coordinata di studi e di ricerche che può procedere per gradi, per singoli fenomeni, per aree di interesse, per singole opere o per autori, ma nel suo insieme potrebbe tessere una trama capace di introdurre, anche dal punto di vista metodologico, nuovi approcci critico-interpretativi portando a una visione più "aperta" della contemporaneità letteraria italiana ${ }^{4}$.

Il punto di confine fra il dentro e il fuori Italia, ma anche fra il prima e il dopo, rispetto alla contemporaneità (quale?), mi pare possa collocarsi dalla metà degli anni Ottanta, quando si demarcano i confini di un passaggio negli studi letterari sia dal punto di vista teorico e storiografico, che dal punto di vista della produzione narrativa e poetica; anni in cui si assiste a decisivi cambiamenti dell'industria libraria con l'acquisizione dei marchi editoriali da parte dei gruppi industriali (Fininvest e RCS per fare solo due esempi). La circolazione dei libri italiani all'estero assume una nuova fisionomia e inizia a maturare un interesse anche in ambito accademico e scientifico per il tema della letteratura italiana all'estero. E intanto l'Europa si prepara al suo nuovo disegno politico e sociale.

In questo contesto di trasformazioni politiche, industriali, culturali dal punto di vista della ricerca letteraria si presenta in Italia una generazione di intellettuali, la generazione dei nati negli anni Trenta, critici militanti, accademici e scrittori, fra cui ricordo Giuseppe Pontiggia (del 1934), Vincenzo Consolo (1933), Umberto Eco (del 1936) e Claudio Magris (del 1939). Fra le loro opere, distanti le une dalle altre, una sola ottiene un indiscusso successo

3. Con questa prospettiva è stato avviato il monumentale progetto dell'Index translationum, sin dagli anni Trenta, nel "decennio delle traduzioni”, gestito dall'Unesco dal 1946 e informatizzato a partire dal 1979, un repertorio delle opere tradotte in tutto il mondo che naturalmente si confronta con la parzialità di informazioni presenti: http://portal.unesco.org/culture/en/ev.php-URL_ID=7810\&URL_DO=DO_ TOPIC\&URL_SECTION=201.html, 15.7.2012. Utile strumento anche Worldcat: https://www.worldcat.org.

4. Classici italiani nel mondo (Italian Classics in the World) è il titolo di una ricerca avviata alla Sapienza Università di Roma, in collaborazione con la Fondazione Arnoldo e Alberto Mondadori BooksinItaly, con prospettive metodologiche diverse: la fortuna all'estero delle autrici ; il "caso" dell'autore del Novecento più tradotto all'estero, Calvino nel mondo, con l'iniziativa internazionale Calvino qui e altrove, la diffusione della letteratura italiana in Svezia (Di Nicola, Laura, Schwartz, Cecilia, Libri in viaggio. Classici italiani in Svezia, cit.) 
internazionale: Il nome della rosa, 1980 (Premio Strega e best-seller anche all'estero) $)^{5}$. Di questa generazione fanno parte anche Francesca Sanvitale (1928), Amelia Rosselli (1930), Rosetta Loy (1931), Dacia Maraini (1936). Si può forse dire che essi, gli "eredi" del passato, insieme agli autori e alle autrici nati negli anni Quaranta e Cinquanta (Vincenzo Cerami, 1940; Sebastiano Vassalli, 1941, Antonio Tabucchi, 1943; Domenico Starnone, 1943; Clara Sereni, 1946; Patrizia Cavalli, 1947; Stefano Benni, 1947; Aldo Busi, 1948; Daniele Del Giudice, 1949) inaugurano una "nuova tradizione" di autori italiani, futuri classici del nuovo millennio, proiettati verso una nuova idea di letteratura? È tutto da discutere.

La generazione di mezzo (Andrea De Carlo, 1952; Patrizia Valduga, 1953; Pier Vittorio Tondelli, 1955; Michele Mari, 1955; Eraldo Affinati, 1956; Marco Lodoli, 1956; Alessandro Baricco, 1958; Sandro Veronesi, 1959) apre la strada a quella dei giovani ventenni che si formano negli anni Ottanta, i nati negli anni Sessanta e Settanta, che Asor Rosa definisce "esploratori del magma" (Carofiglio, 1961, Margaret Mazzantini, 1961, Luigi Guarnieri, 1962, Tiziano Scarpa, 1963, Francesco Piccolo, 1964, Antonio Manzini, 1964; Diego De Silva, 1964; Giuseppe Culicchia 1965; Elena Stancanelli, 1965, Niccolò Ammaniti, 1966, Melania Mazzucco, 1966, Aldo Nove, 1967, Marco Giovenale, 1969; Silvia Ballestra, 1969, Laura Pugno, 1970, Simona Vinci, 1970, Giorgio Vasta, 1970; Michela Murgia, 1972; Nicola Lagioia, 1973; Valeria Parrella, 1974, Mario Desiati, 1977). Del 1979 è Roberto Saviano che occupa uno spazio particolare, introducendo i nati negli anni Ottanta, Paolo Giordano (1982), Paolo Di Paolo (1983), Silvia Avallone (1984) ${ }^{6}$.

Questi elenchi di nomi definiscono, oltre a tendenze, fenomeni, sperimentazioni (che in questa sede non è possibile approfondire), tre diverse generazioni di narratori e poeti, con caratteristiche e specificità stilistiche, tematiche e linguistiche le più differenziate ma che segnano il panorama letterario italiano dell'ultimo trentennio. Una scelta di opere "classiche" risulterebbe arbitraria se non motivandola caso per caso; tanto più in un momento in cui l'industria libraria

5. Si veda Tarabbia, Andrea, "Quando la cultura conquista il mondo. Dai Premi Nobel a Umberto Eco", in Copy in Italy. Autori italiani nel mondo dal 1945 a oggi, a cura di Fondazione Arnoldo e Alberto Mondadori, Milano, Effigie, 2009, pp. 158-163.

6. Su alcune questioni che ciascun "gruppo" di autrici e autori pone si veda: BARENGHI, Mario, Oltre il Novecento. Appunti su un decennio di narrativa (1988-1998), Milano, Marcos Y Marcos, 1999; Asor RosA, Alberto, "Scrittori e massa. Saggio sulla letteratura italiana postmoderna”, in ID., Scrittori e popolo. 1965. Scrittori e massa. 2015, Torino, Einaudi, 2015, pp. 355-422. 
diventa protagonista delle selezioni che si operano non dentro un sistema letterario, quanto piuttosto dentro un sistema di vendite. Gli autori sono destinati per il momento a stare dentro le classifiche dei più venduti, più letti, più tradotti, più conosciuti, segnati dall'appartenenza a un editore, una collana, un'antologia, in attesa di passare dalle "classifiche" verso un "canone" che esprima valori letterari universali.

$\mathrm{Al}$ momento l'unica operazione possibile, oltretutto ancora empirica, e piuttosto paradossale, sembra quella di cominciare a individuare gli autori di oggi che nel mondo più rappresentano la letteratura di questo ultimo quindicennio. Nomi che definiscono un canone che dall'estero si riflette verso l'Italia e con cui l'Italia stessa si misura. D'altra parte è vero che, viceversa, nelle scelte operate dal "fuori Italia" possono incidere fattori come i riconoscimenti nazionali, $i$ premi letterari ottenuti nel paese d'origine. In questa prospettiva si osserva che il premio Strega in questa ultima fase, in cui la critica letteraria ha ceduto il passo all'editoria nella "costruzione" dei classici del Novecento ${ }^{7}$ svolge un ruolo di primo piano nella fortuna della narrativa italiana fuori d'Italia ${ }^{8}$. I titoli che trovano un maggiore riconoscimento sono infatti libri che hanno vinto il Premio Strega: Caos Calmo di Veronesi, Non ti muovere di Margaret Mazzantini, Come Dio comanda di Niccolò Ammaniti, Vita di Melania Mazzucco. Per i più giovani occorre citare La solitudine dei numeri primi, del 2008 e Acciaio del 2012. A questo gruppo di opere vincitrici del premio Strega, bisogna aggiungere, per definire le opere recenti più diffuse nel mondo, Gomorra entrato come best seller nei mercati internazionali, tradotto in 52 paesi, e il caso Ferrante. Gli autori contemporanei più tradotti all'estero, pur nella provvisorietà delle indagini, sembrano essere Ammaniti, Mazzantini, Giordano, Avallone, Ferrante, Mazzucco. Sono tutti ampiamente diffusi e conosciuti in Europa, mentre in altre lingue osserviamo diversità di scelte: in greco sono tradotti Mazzucco e Mazzantini; in Vietnam Mazzucco, Ammaniti e Giordano; in Cina Ammaniti, Giordano e Mazzantini; in arabo Ferrante, Giordano, Avallone. Saranno loro i futuri classici italiani del terzo millennio nel mondo?

Nei processi di diffusione degli autori italiani contemporanei all'estero i fattori trainanti sono molteplici e diversificati. Possono incidere oltre al riconosci-

7. Si veda su questo importante aspetto Cadioli, Alberto, "L'editoria nella 'costruzione' dei classici del Novecento", in Di Nicola, Laura, Schwartz, Cecilia, Libri in viaggio. Classici italiani in Svezia, cit., pp. 91-99.

8. Per un'analisi su questo tema rimando a una recente indagine di Lucia Diletta Capuano, La fortuna della narrativa italiana all'estero: il caso del premio Strega, tesi di laurea, relatore prof. Giovanni Solimine, Sapienza Università di Roma, a.a. 2015-2016. 
mento, alla notorietà e al successo nazionale, il peso dell'editore italiano, dell'agente letterario (Wylie è l'agente di Baricco, Magris, Saviano, Giordano; Carpinelli di Ammaniti, Mazzantini, Mazzucco, per fare qualche esempio) e, direi, un certo nomadismo e cosmopolitismo culturale. Il fenomeno dell'emigrazione di autori e autrici italiani all'estero si declina in forme diverse: scrittori italiani che nascono all'estero (Mazzantini nasce a Dublino), che vi soggiornano per brevi o lunghi periodi, l'attitudine a conoscere lingue diverse dalla propria, un poliglottismo diffuso che favorisce anche le presentazioni della propria opera nel mondo e della cultura del nostro paese. Gli scrittori e le scrittrici di oggi svolgono un ruolo centrale anche come promotori culturali, nella percezione della cultura e della lingua italiane nel mondo: sono coloro che portano fuori d'Italia la narrazione dell'Italia presente, una nuova letteratura che procede parallela al recupero dei classici del passato.

A questi fattori più intrinseci possono subentrare anche fattori esterni per definire lo sguardo del "fuori", primo fra tutti quello più difficile da indagare ed esplorare: l'occasionalità o, forse sarebbe meglio dire, il caso, che può essere indagato su singoli episodi e circostanze.

Torniamo ancora una volta a esprimere che lo snodo storiografico è lì, negli anni Ottanta. Si afferma in ambito internazionale Umberto Eco; nasce la più giovane generazione di autori di oggi, Giordano, Di Paolo, Avallone; le riviste letterarie, sede centrale di dibattito nel corso del secolo, entrano in crisi; scompare un'intera generazione di scrittori e scrittrici italiani (oltre Calvino, Primo Levi, Morante, Sciascia, Moravia, Ginzburg) cui guarda l'editoria internazionale; la questione della fortuna dei classici italiani all'estero diventa un tema di confronto fra l'italianistica in Italia e fuori Italia ${ }^{9}$. Quasi contestualmente, fra il 1987 e il 1988, escono i volumi della Letteratura italiana diretta da Asor Rosa dedicati alla Storia e geografia prefigurando nuove ipotesi per interrelare il sistema letterario.

Nel 1985, inoltre, proprio a delimitare un prima e un dopo, muore, all'età di 61 anni, Italo Calvino, l'autore più tradotto fuori Italia (in 65 paesi, in 57 lingue), l'ultimo dei classici, secondo Asor Rosa, di quella generazione di autori nati negli

9. L'interesse scientifico per la questione si fa esplicito, infatti, proprio nel $1988 \mathrm{con}$ due convegni (uno in Olanda e l'altro in Italia): Lo CAscio, Vincenzo (a cura di), Lingua e cultura italiana in Europa, Atti di congresso (Amsterdam, ottobre 1988), Firenze, Le Monnier, 1990; BALdelli, Ignazio, DA Rif, Bianca Maria (a cura di), Lingua e letteratura italiana nel mondo oggi, Atti del XIII Congresso dell'Associazione internazionale per gli studi di lingua e letteratura italiana (Perugia, 30 maggio - 3 giugno 1988), Firenze, Olschki, 1991. 
anni Venti (fra cui ricordiamo Meneghello, Fenoglio, Pasolini) che chiudono una fase della storia letteraria del Novecento (mentre il coetaneo Camilleri, nato nel 1923, sembra appartenere, per il tardivo esordio letterario, alla fase più recente attraversando pienamente il nuovo millennio).

Proprio nel 1985, a quindici anni di distanza dall'inizio del nuovo millennio e poco oltre trent'anni da oggi, Calvino con i Six memos for the next millennium stabilisce un ponte fra la letteratura del passato e il futuro della letteratura nel celebre inizio: "Siamo nel 1985: quindici anni appena ci separano dall'inizio d'un nuovo millennio". Un futuro al quale si può guardare a partire dal millennio passato che "ha visto nascere ed espandersi le lingue moderne dell'Occidente e le letterature che di queste lingue hanno esplorato le possibilità espressive e cognitive e immaginative"; ed "è stato anche il millennio del libro, in quanto ha visto l'oggetto-libro prendere la forma che ci è familiare"10. Il discorso di Calvino mette al centro le lingue, le letterature e il futuro del libro, i fondamenti conoscitivi per interrogare l'Italia fuori Italia, in Europa, nell'Occidente, nei riflessi dell'Oriente, nelle prospettive che la attendono; per proiettare la letteratura italiana in un'idea di letteratura universale, mondiale, d'ogni tempo e paese, popolare e colta.

Per primo Calvino propone un "canone", parola che l'autore non utilizza mai nelle sue conferenze, ovvero una scelta, una selezione, non tanto di classici, autori e opere, quanto piuttosto di valori, di qualità permanenti, un canone interiore, soggettivo, singolare e individuale, che esprime un'idea della letteratura, della sua funzione etica, esistenziale e conoscitiva. Il permanere universale non è nelle opere ma nei valori di cui sono portatrici. Nelle Lezioni americane Calvino non usa mai la parola "classico" (solo indirettamente, attraverso una citazione di Raymond Queneau a proposito della libertà di seguire regole e della schiavitù dell'ispirazione "Le classique qui écrit sa tragédie en observant un certain nombre de règles qu'il connaît est plus libre que le poète qui écrit ce qui lui passe par la tête et qui est l'esclave d'autres règles qu'il ignore") ${ }^{11}$. Calvino preferisce la parola "tradizione" come campo di tensioni fra il prima e il dopo, fra culture letterarie diverse, fra saperi, generi letterari, esprimendo la sfaccettata pluralità delle diverse prospettive (per esempio, a proposito della concezione atomistica della scrittura di Lucrezio, la colloca come inizio di "una lunga tradizione di pensatori"; la leggenda di Carlomagno "ha dietro di sé una tradizione nella letteratura italiana"; "la tecnica della narrazione orale nella tradizione popolare"; "La

10. Calvino, Italo, Lezioni americane (1988), Milano, Mondadori, 1993, p. 7. 11. Ibid., p. 134. 
letteratura americana ha una gloriosa tradizione di short stories tuttora viva"; $L a$ vie mode d'emploi di Georges Perec come "compendio d'una tradizione narrativa") ${ }^{12}$. $\mathrm{Al}$ "classico", Calvino, come è noto, aveva dedicato qualche anno prima, nel 1981 (ricordo che nel 1978 Franco Fortini aveva pubblicato la voce nell'Enciclopedia Einaudi), un articolo sull'Espresso, ampiamente citato per gli usi più diversi, Italiani vi esorto ai classici, poi con il titolo d'autore Perché leggere i classici, scelto per introdurre, a distanza di dieci anni, la raccolta postuma del $1991^{13}$. Calvino in quell'occasione, argomentando le quattordici proposte di definizione, pone in maniera implicita la questione del rapporto con la tradizione, con il canone, con i classici: "leggere i classici sembra in contraddizione $[\ldots]$ con l'eclettismo della nostra cultura che non saprebbe mai redigere un catalogo della classicità che fa al caso nostro" e aggiunge: "I vecchi titoli sono stati decimati ma i nuovi sono moltiplicati proliferando in tutte le letterature e le culture moderne. Non resta che inventarci ognuno una biblioteca ideale dei nostri classici"1", cosa che lui stesso farà con le Lezioni americane. Il "catalogo della classicità" è un'operazione che non può appartenere alla nostra cultura, Calvino propone piuttosto un "canone", diremmo noi, come ordine di qualità, valori permanenti che corrispondono a un campo di tensioni individuale: "Nell'universo infinito della letteratura s'aprono sempre altre vie da esplorare, nuovissime o antichissime, stili e forme che possono cambiare la nostra immagine del mondo..." ${ }^{15}$. Fra nuovo e antico, fra classico e moderno, la lezione di Calvino, l'ultimo dei classici, si offre come un ponte fra i classici moderni, contemporanei, e i classici del futuro.

Il Calvino teorico, con una vena critica nei confronti del mondo accademico, pone questioni sul rapporto con la tradizione che anticipano il dibattito sui "Classici" e sul "Canone", che ha segnato tutto l'ultimo trentennio, per mettere in primo piano lo sguardo, l'individualità, la soggettività del lettore e, aggiungo, dell'interprete: nel 1985 era arrivato allo stesso punto cui siamo giunti oggi, dopo aver attraversato decenni di discussioni teoriche, metodologiche sui classici e sul canone (l'anti-canone, canone liquido, canone mobile).

Negli anni Ottanta altri fattori incidono sulla costruzione del ponte che porta la letteratura verso il nuovo millennio: nel 1982 inizia un'impresa critica, storio-

\section{Ibid.}

13. Calvino, Italo, Italiani, vi esorto ai classici (1981), poi in ID., Perché leggere i classici, Milano, Mondadori, 1991, pp. 11-19.

14. Ibid., p. 19.

15. Calvino, Italo, Lezioni americane, cit., p. 11. 
grafica, teorica, di grande importanza per l'italianistica, la Letteratura Italiana Einaudi, che prosegue in 16 volumi fino al 2000 (e più recentemente ristampata con revisioni e aggiornamenti, dalla Repubblica in 23 volumi, dal settembre 2007 al febbraio 2008). Un'opera che accompagna quindi le riflessioni sulla letteratura italiana, sulla tradizione e la ricerca letterarie, sui metodi di analisi, sulla definizione del canone, dagli anni Ottanta a oggi e sulla quale si è formata una generazione di studiosi che oggi occupano lo spazio della critica (se ancora esiste) e della formazione universitaria. Il problema del metodo (che lo stesso Asor Rosa comincia a porre proprio in quella data del 1985, Metodo e non metodo nella critica letteraria) sembra ignorato dalla ricerca umanistica. L'italianista, oggi, allora ha il compito di garantire "al massimo grado la persistenza dei valori umanistici, non ideologicamente, e perciò vanamente, rivendicati, ma puntigliosamente ritrovati, riga per riga, capoverso per capoverso, canto per canto, autore per autore, nel cumulo di eredità che il passato, fortunatamente, ancora ci consegna" 16 .

Non intendo riattraversare quel dibattito scientifico e non, dentro e fuori l'accademia, al quale hanno partecipato scrittori, intellettuali, critici, preceduti da Eliot, Fortini ${ }^{17}$, prima di arrivare a Calvino. Certo è che dopo la raccolta postuma curata da Esther Calvino Perché leggere i classici del 1991, l'anno seguente, nel 1992, Asor Rosa per primo affronta il tema del canone con Il canone delle opere (1992), cui segue il celebre saggio del critico americano Bloom The Western Canon $(1994)^{18}$. I due diversi, antitetici, punti di vista, di Asor Rosa e Bloom, segnano le premesse del vivace dibattito cui prendono parte Curi ${ }^{19}$, Luperini, Rivoletti, Jauss, Battistini, Ceserani, Ferroni, Guglielmi, Pasero, Segre ${ }^{20}$. Si iniziano a prefigurare e a intrecciare, negli anni Novanta, alcune altre grandi questioni: l'italiano all'estero (Riflessioni sul canone della letteratura italiana nella prospettiva

16. Asor Rosa, Alberto, Letteratura italiana. La storia, i classici, l'identità nazionale, Roma, Carocci, 2014, p. 10.

17. Eliot, Thomas Stearns, What Is a Classic?, 1944. (tr. it. "Che cos'è un classico?", in Sulla poesia e sui poeti, Milano, Garzanti, 1960, pp. 55-75); Fortini, Franco, Classico, in Romano, Ruggero (a cura di), Enciclopedia, vol. 3, Torino, Einaudi, 1978, pp. 192-202.

18. Asor Rosa, Alberto, Il canone delle opere, in ID. (a cura di), Letteratura italiana. Le opere, Vol I. Dalle Origini al Cinquecento, Torino, Einaudi, 1992, pp. XXIII-LV; BLOOM, Harold, The western canon, New York, Harcourt Brace, 1994 (trad. it., Il canone occidentale. Milano, Bompiani, 1999). Il rapporto fra i due testi, le distanze e le affinità, è discusso dallo stesso Asor Rosa: Asor Rosa, Alberto, Il tempo dei classici (1998), in ID., Letteratura italiana. La storia, i classici, l'identità naz̧ionale, cit., pp. 120-121.

19. Curi, Fausto, Canone e anticanone. Studi di letteratura, Bologna, Pendragon, 1997.

20. Sul canone, numero speciale della rivista Allegoria, n. 29-30, maggio-dicembre, 1998. 
dell'insegnamento all'estero") ${ }^{21}$, la crisi della critica letteraria ${ }^{22}$, la contestuale attenzione verso lo studio dei fenomeni letterari in prospettiva editoriale (Garin, Turi, Ragone $)^{23}$, l'affermarsi degli studi di genere (Zancan, 1998) ${ }^{24}$ : aspetti di cui bisogna tener conto per un'analisi del fenomeno della diffusione della letteratura italiana all'estero.

Gli anni Novanta definiscono le premesse, insomma, di quel dibattito sul canone che matura negli anni Duemila, attraverso volumi collettanei e non che esprimono la necessità di definire una selezione fra le opere del Novecento, una prospettiva metodologica e teorica per operarla. Si tratta, fra gli altri, di sei volumi usciti in un paio d'anni: Il canone letterario del Novecento italiano (2000); Il giudizio di valore e il canone letterario (2000); Il Canone alla fine del millennio (2000); Il canone letterario (2001); Un canone per il terzo millennio (2001); Il canone e la biblioteca. Costruzioni e decostruzioni della tradizione letteraria italiana (2002) ${ }^{25}$. È in questo contesto di indagini che il tema della letteratura italiana all'estero entra fra le questioni storiografiche $e^{26}$.

A partire dagli anni Dieci, la direzione degli studi letterari, attenta a nuovi fenomeni socio-culturali, agli studi post-coloniali, al problema delle scrit-

21. Numero della rivista Quaderns d'Italia, n. 4/5, 1999-2000 con interventi di Antonelli, Ceserani, Coletti, Di Girolamo, Ferroni, Luperini, Spinazzola, Colsar.

22. Cfr. Segre, Cesare, Notizie dalla crisi. Dove va la critica letteraria?, Torino, Einaudi, 1993. Ganeri, Margherita, Merola, Nicola (a cura di), La critica dopo la crisi. Atti del Convegno di Arcavacata, 11-13 novembre 1999, Soveria Mannelli, Rubbettino, 2002. Ricordo anche SEgre, Cesare, Ritorno alla critica, Torino, Einaudi, 2001; ID., Critica e critici, Torino, Einaudi, 2012.

23. Garin, Eugenio, Editori italiani fra Ottocento e Novecento, Roma-Bari, Laterza, 1991; Turi, Gabriele (a cura di), Storia dell'editoria nell'Italia contemporanea, Firenze, Giunti, 1997; Ragone, Giovanni, Un secolo di libri. Storia dell'editoria in Italia dall'Unità al postmoderno, Torino, Einaudi, 1999.

24. ZANCAN, Marina, Il doppio itinerario della scrittura. La donna nella tradizione letteraria italiana, Torino, Einaudi, 1998; EAD., "Letteratura, critica, storiografia. Questioni di genere", Bollettino di italianistica, vol. 4, 2005, pp. 5-31.

25. Merola, Nicola (a cura di), Il canone letterario del Novecento italiano, Soveria Mannelli, Rubbettino, 2000; InNOCENTI, Loretta (a cura di), Il gindizio di valore e il canone letterario, Roma, Bulzoni, 2000; EAD., "Il Canone alla fine del millennio", Critica del testo, a. III/1, 2000; Onofri, Massimo, Il canone letterario, Roma-Bari, Laterza, 2001 e, lo stesso anno, Olivieri, Ugo M. (a cura di), Un canone per il terzo millennio. Testi e problemi per lo studio del Novecento tra teoria della letteratura, antropologia e storia, Milano, Bruno Mondadori, 2001; Quondam, Amedeo (a cura di), Il canone e la biblioteca. Costruzioni e decostruzioni della tradizione letteraria italiana, Roma, Bulzoni, 2002.

26. Cfr. D’Intino, Franco, Il Novecento italiano oltrefrontiera, cit., pp. 917-995; ForMISANO, Luciano (a cura di), La letteratura italiana fuori dall'Italia, in Storia della letteratura italiana, a cura di Enrico Malato, vol. 12, Roma, Salerno, 2002. 
ture migranti, va proiettando l'italianistica oltreconfine in una visione "globale" della letteratura per ridiscutere il concetto di canone, di tradizione, e di identità nazionale ${ }^{27}$, sentendo la necessità di riconsiderare la parola "classico" 28 . In anni recenti, Ossola intitola Canone dei classici gli otto volumi della Letteratura italiana pubblicati per la UTET ${ }^{29}$, mentre Asor Rosa ritorna alla sua prima impresa degli anni Ottanta con una raccolta di saggi dal medesimo titolo Letteratura italiana cui aggiunge significativamente il sottotitolo La storia, $i$ classici, l'identità naz̧ionale. Fra storia e identità nazionale si ripone la questione dei "classici". A distanza di vent'anni, il discorso per Asor Rosa assume una nuova prospettiva: "Quando i 'classici' - così come li intendo io, scrive nel 2014 -, invece di adoperarsi a continuare a rafforzare la tradizione, la sovvertono, calandosi nelle profondità della psiche e dell'esperienza umana, allora qualcosa di nuovo comincia: magari una 'nuova tradizione', diversa da quella passata anche se ad essa (quasi sempre collegata)"30.

Non si può dunque parlare di una "nuova tradizione" se essa non si collega alla Tradizione del passato, i classici del futuro troveranno il permanere dei loro valori se radicati nei classici del passato. Il rapporto con i classici, plurimo, ramificato, si confronta con i fattori più diversi, con i tempi dell'uomo e della società; con la scelta, la selezione, le memorie individuali, collettive e culturali; con una prospettiva mai nazionale ma sempre europea o occidentale, che tenga conto però dei riflessi delle culture orientali.

Il problema della "costruzione" del canone e del "classico" nel confronto fra prospettiva nazionale e internazionale, fra generazioni di autrici e autori, fra $i$ classici di oggi e quelli di domani, ci pone di fronte all'ossimoro dei "classici contemporanei" nel mondo, il cui futuro anteriore degli anni Zero sempre più si sposta verso la tradizione, verso il passato in un canone, storico o individuale, che risponde ai criteri più diversi e che si modifica nel tempo.

La proiezione di un canone del nuovo millennio è un canone provvisorio, aperto, plurale, discutibile, liquido, inverso, forse impossibile; ciò che interessa l'italianistica degli ultimi decenni è ciò che resta "fuori" dall'Italia, ma anche "fuori" dal canone inteso come tradizione letteraria, storiografia. Il processo di

27. Coletti, Vittorio, Romanzo mondo. La letteratura nel villaggio globale, Bologna, Il Mulino, 2011; Benvenuti, Giuliana, Ceserani, Remo, La letteratura nell'età globale, Bologna, Il Mulino, 2012.

28. Cfr. Tatтi, Silvia, Classico. Storia di una parola, Roma, Carocci, 2015.

29. Ossola, Carlo (a cura di), Letteratura italiana. Canone dei classici, 8 voll., Torino, Utet, 2012.

30. Asor Rosa, Alberto, Letteratura italiana. La storia, i classici, l'identità nazionale, cit. 
canonizzazione di oggi è quello che fa entrare dentro ciò che è rimasto ai margini della storia. Fuori sono rimaste a lungo le opere delle autrici, fuori la letteratura della migrazione (che le nuove tendenze critiche stanno riposizionando) e i quadri storiografici esprimono tutta la loro provvisorietà di cui dobbiamo tener conto (anche nell'insegnamento).

Per rispondere alla domanda: quale canone per il nuovo millennio? Forse semplicemente si può riprendere la lezione di Calvino e riconoscere e riconoscersi nel canone dell'interiorità, di quei classici contemporanei che si accostano negli scaffali di ciascun lettore senza un preciso ordine, né cronologico, né geografico, ma che hanno insegnato a ciascuno, in modo diverso, a leggere il mondo. Perché alla fine la risposta alla domanda cruciale è questa - scrive Melania Mazzucco, una "classica" del futuro:

Cosa è universale e permanente? Tutto ciò che riguarda l'essenza delle cose - non la loro apparenza. Il corpo della letteratura, non il suo vestito. I nervi, la pelle, i muscoli e il sangue. Le storie degli uomini e delle donne, le cose di tutti $i$ giorni e di sempre, le sole forse importanti. I sentimenti, le idee, gli amori, la morte, le speranze, le delusioni, i dolori, le gioie - la vita, insomma. Queste parlano a tutti, ovunque siano nati e in qualunque lingua ci stiano leggendo. Anche senza metafore, senza gerghi, senza capriole stilistiche e perfino senza la musica delle parole incatenate. Alla fin fine, i romanzi che amiamo parlano proprio della vita. Essi possono viaggiare ovunque - ma senza bagaglio: nudi e leggeri ${ }^{31}$.

Laura Di NicolA

Sapienza - Università di Roma

31. Mazzucco, Melania G., "Briciole sulla traduzione", in Copy in Italy. Autori italiani nel mondo dal 1945 a oggi, cit., p. 214. 